\title{
A Review on Inter-Satellite Links Free Space Optical Communication
}

\author{
Gayatri Tiwari* and Ram Chandra Singh Chauhan \\ Department of Electronics Engineering, Institute of Engineering \& Technology, \\ Lucknow, India
}

\begin{abstract}
Article Type: Article
Article Citation: Gayatri Tiwari, Ram Chandra Singh Chauhan. A review on inter-satellite links free space optical communication. Indian Journal of Science and Technology. 2020; 13(06), 712-724. Dol: 10.17485/ijst/2020/ v013i06/147998
\end{abstract}

Received date: 0ctober 5, 2019

Accepted date: January 2, 2020

*Author for correspondence: Gayatri Tiwari gayatri.10nov@ gmail.com 9 Department of Electronics Engineering, Institute of Engineering \& Technology, Lucknow, India

\begin{abstract}
Objectives: The need for constricting, lightweight, low-power electronics-aggravated by the thriving demand for large data flow rate and bandwidth is urging the use of optical technologies in defense and space. Free space optical (FSO) communication links have promising eminence over microwave communication links with high speed, low weight, and increased security so that they can be boarded conveniently on the satellite. Methods/findings: The advancement of the optical components and systems has made it very predominant along with its acceptability in space and defense. Yet a couple of significant components to be dealt with are tracking problems with misalignment of the transmitter and receiver apertures and the progressions because of barometrical conditions. Efficiency of the FSO communication system is seriously rotted due to the angle of arrival (AoA) variance and including error pointing. The optical transmitter can be perfectly coordinated with the receiver by utilizing the pointing, acquisition, and tracking (PAT). However, this method is to be carried out under the airborne platform with required restrictions namely size, weight, and power. Application: An overview of promising inter-satellite applications with the challenges have been discussed which sway the performance of the system and make the communication link unattainable. The FSO communication possesses an advantage over two existing technologies optical fiber communication and wireless communication. Hence, the significant use of FSO is intersatellite optical wireless communication (Is OWC) links, which will be deployed in the future in space.
\end{abstract}

Keywords: Angle-of-arrival Variance (AoA), Free-space Optical Communication, Pointing, Acquisition, and Tracking (PAT)

\section{Introduction}

Ongoing years have seen enormous development in the field of information and communication techniques. The majority of this becomes conceivable with progression and 
utilization of light wave technology in the field of communication. With fast, information transmission over larger distance has turned out to be conceivable [1]. This offered ascend to the optical wireless communication (OWC) being connected economically. Because of numerous points of interest over custom radio frequency signal (RF), OWC has been as of now connected in number of uses. LASER has been utilized seriously because of their coherent properties and in this way the equivalent is utilized to transmit information in space to convey between satellites orbiting in same or different orbits. This innovation has been named as inter-satellite optical wireless communications (Is-OWCs) [2]. With properties like rapid and high limit, Is-OWC system empowers progressively successful, productive and steady activity of satellite system in years to come [3]. In [4-5] this way by utilization of Is-OWC, a transformative space system can be shaped from satellites which can fill in as both backbone as well as user access nodes.

The first inter-satellite links utilized microwaves radio frequency (RF ISL) worked under Ka-band frequency groups which prone to be helpful for mobile satellite system (MSS). It is helpful in MSS for example, Iridium Next and Artemis, however, with the quick improvement of satellite interchanges which requires a massive bit rate [6], further to proceed towards the optical ISLs is important as it is referred to by their focal points, for example, high information rate, an enormous transfer speed, long communication distance, minute transmission power with improved reliability and being cost effective, small antenna diameter and, more reliability and information security. Likewise, it is simple for multiplexing, DE multiplexing, exchanging, and directing adaptability of the system applications. The primary optical ISL between satellites utilizing laser light was set up between the European Space Agency's (ESA) and the French agency possessing Artemis satellite and the SPOT-4 an Earth perception satellite was the primary optical ISL utilizing laser light under the guidance of an experiment called Semiconductor Laser Inter satellite link experiment [7-8]. For optical ISLs, three specialized parameters are important to set up an association between satellites; initial one is the frequency bands where it is in the scope of Tera Hertz (THz) to accomplish huge information rate (Table 1). The fundamental thought of transmission of the signal exposed to the free space experiences loss since zero absorption occurs in the signal. Reason to this loss is the nonalignment of the atmosphere.

And the second one is a technique named as multiple access which is used for allocating the capacity of satellite transponder for the interference escaping among the approaching signal via satellites for the concerned earth stations [9], station limit is $\mathrm{C}=\mathrm{B} \log _{2}(1+$ $\mathrm{S} / \mathrm{N})$, where "B" refers to the transfer speed, and $\mathrm{S} / \mathrm{N}$ symbolizes ratio of signal to noise

TABLE 1. Optical frequency and transmitted power

\begin{tabular}{lll}
\hline Types of laser & Wavelength $(\boldsymbol{\mu m})$ & T transmitted power \\
\hline Solid state laser diode & & \\
ALGaAs & $0.8-0.9$ & About $100 \mathrm{~mW}$ \\
InPAaGa & $1.3-1.5$ & About $100 \mathrm{~mW}$ \\
$\mathrm{Nd}$ YAG & 0.532 & $100 \mathrm{~mW}$ \\
Gas laser & & \\
$\mathrm{CO}_{2}$ & 10.6 & Several tens of watts \\
\hline
\end{tabular}


ratio. Thus, the multiple wavelengths are utilized for the increment in the system limit at the time of demand approaching towards the final extent of single optical channel [10].

The method of modulation is used which is supposed to be critical for the optimum modulation format [11] regarding a most extreme theoretical limit. This idea gives additionally phenomenal security which increases the immunity against jamming and interception [12]. The concerning point of this particular paper is to review about the Is OWC, which transfers the data and collects the information among the satellites, and detailed examination with clarification of the need to choose optical transmission. A review on different difficulties looked by free space optical (FSO) communications system for inter-satellite links has been deliberated. Section I presents the brief introduction on IS-OWC system. While Sections II discusses some related work and explains the different types of challenges for inter-satellite links confront by FSO communication system .Section III discusses the advantages of IS-OWC over RF communication system. Last section is a conclusion and gives future perspective for the optical inter-satellite links.

\section{Is-OWC System}

Communication between two satellites is represented in Figure 1. Whereas, Figure 2 describes the coverage of the Earth's surface area by the inter satellite laser links.

By free space optics, signal is transmitted between two satellites in Is-OWC. Block diagram of the Is-OWC represented by Figure 3 [13-14].

\subsection{Transmitter}

LASER is a source which produces light and it is carried by transmitting satellites, as well as used for tracking, satellite telemetry, for communication system and an optical modulator [15]. The information which is regularly transmitted by a satellite is same as the zone of the satellite and following of attitude, picture getting the data for which transmission is done

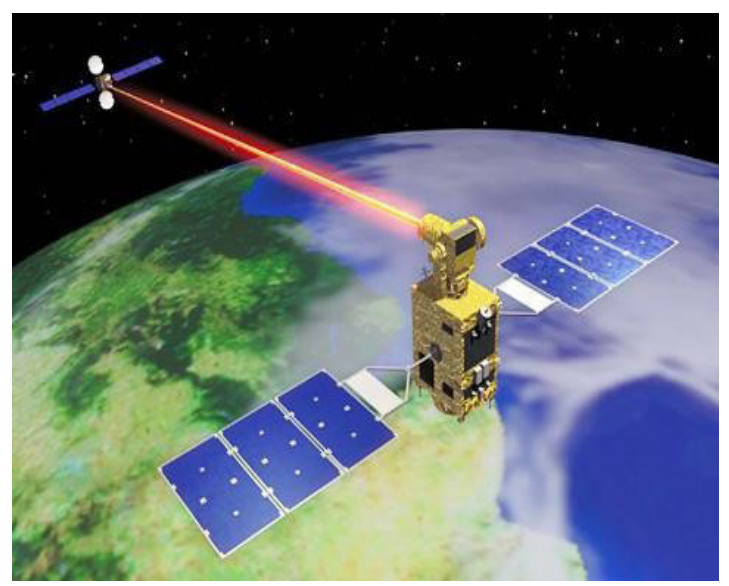

FIGURE 1. Inter-satellite optical communications. 


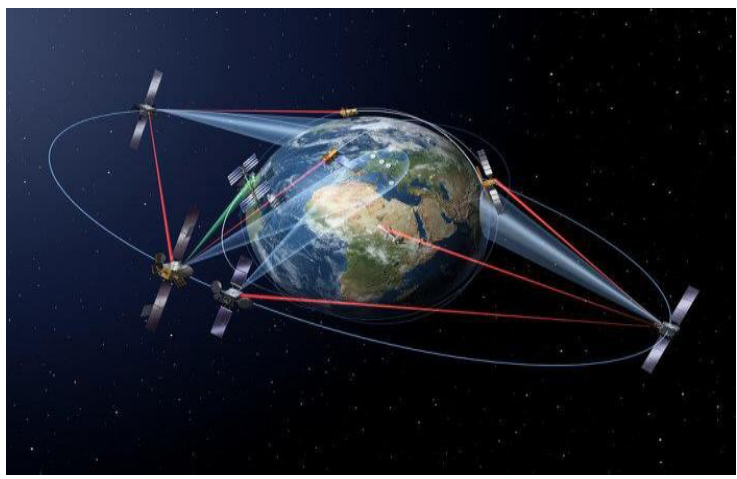

FIGURE 2. Inter-satellite laser links around the Earth.

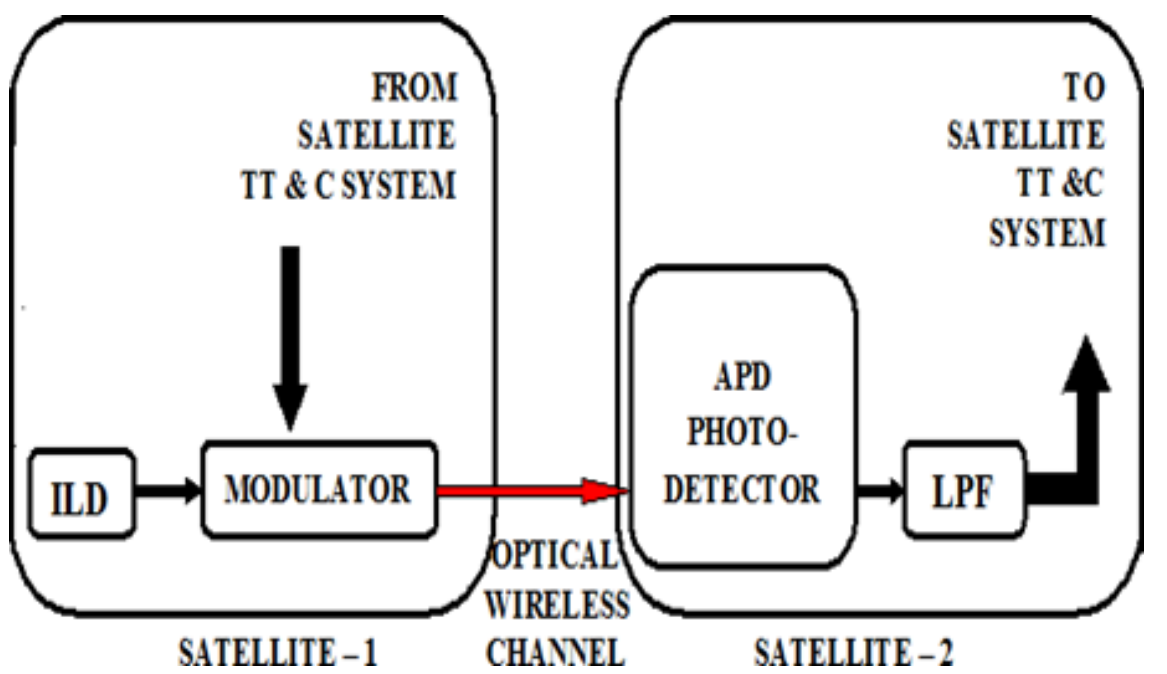

FIGURE 3. Basic system block diagram for simplex communication in Is-OWC.

by a satellite are, for instance, the satellite territory and atmosphere following for isolated distinguishing satellite and communicating data got ready for handset system passing on satellite. The most liberal portion in the structure is light source as correspondence is done by transmitting light. Two sorts of optical light sources are used in optical correspondence, i.e., light-emitting diode (LED) and injected laser diode (ILD). Development of these devices is done by semiconductor materials with the relationship of positive and negative charges semiconductor yielding photons or light imperativeness. Radiation of light from ILD monochromatic, sound and has over the top vivacity making it fitting for covering the colossal division for transmitting in free space [16-17].

\subsection{Propagation Medium}

Considering the condition of Is-OWC framework, the optical remote channel is viewed as the transmission medium. OWC channel is vacuum and free from losses like climatic losses in opposition to free space optics which is acquainted with a few losses attributable 
to climate and air debilitation [18]. Taking the perfect case, transmitting distance is the particular origin for signal attenuation. At the transmitter and the beneficiary side, utilization of optical antenna or optical lenses is formed. Broad divergence of light beam acceptance is allowed by the optical antenna.

\subsection{Receiver}

The signal on the recipient side of the Is-OWC incorporates a photodiode and a low pass channel. The detected received signal is accepted by photodiode that changes it into an electrical signal [19]. Photodiode involves positive and negative charged semiconductor connection, connected in reverse bias simply like in an optical light source [20-21]. Photons strike the junction then only an electrical signal is produced. Since having peculiar features as large amplification of weak light signals in FSO transmission, APD is used [22].

\section{Challenges in Inter-Satellite Links Free Space Optical Communication}

Atmospheric impacts which affect the result of communication between ground to satellite and satellite to ground. FSO innovation utilizes environmental window to breach having characteristics arbitrary as similar to time and space. For inter-satellite FSO links, different constraining elements incorporate availability of link, surround noise, and pointing. The respective paper encompasses various difficulties experienced by data designers with respect to laser uplink/downlink just as inter-satellite FSO links. FSO links are not exposed to the climatic changes or the disturbances because of the clouds since the satellite orbits are placed distant from the atmosphere. For such situation, real test was performed using the acquisition and tracking of the respective two satellites moving at a considerable relative speed. Since large distances have to be covered by the inter-satellite, hence the strategy of the transmission should be of high power efficiency with relatively good sensitivity on the recipient part.

Thus the homodyne or heterodyne being the techniques of the coherent phase are placed convenient over direct detection methods at the inter-satellites FSO links. At the resultant of view, the above techniques present remarkable sensitivity at the receiver end delivered at high capacity links [23-24]. Considering in today's time, the most elevated transmission in between LEO and LEO at a high speed of $5.6 \mathrm{Gbps}$ is the homodyne BPSK transmission. The European Data Relay Satellite System (EDRS) was created by the renowned European space agency which effectively exhibited interface among Alphasat in GEO and Sentinel-1 in LEO at $1.8 \mathrm{Gbps}$ in 2014 [25]. A detailed examination of the elements of the intersatellite FSO links were expected for $1000 \mathrm{~km}$ separation at a speed of $2.5 \mathrm{Gbps}$.On the contrary, knowing the fact that there was no exposure of the FSO links to the climates and air restrictions. The Doppler shift, background radiations, acquisitions, Point ahead angle, tracking, and satellite platform stability were responsible to be the difficulties for any constraints to the FSO for every case. These difficulties are talked about as pursues. 


\subsection{Point Ahead Angle}

The comparative movement between the transmitter and receiver terminals requires the arrival signal to offset from the beacon location with the goal to adequately hit the recipient upon an appropriate location known as a spatial temporal location. The pointing of the offset is called PAA. The time taken to travel for the long distances of cross link is dependent on the comparative speed of the two satellites. The degree of the PAA is of hundreds of micro radians considering space optical links where as it is normally many miniaturized scale radians for the satellite to satellite and ground to satellite. Figure 4 delivers the idea of PAA sharing the optical beam sent by LEO and time " $\mathrm{T}$ " and arrival beam from GEO at " $\mathrm{T}+4 \mathrm{~T}$ ". So for exact pointing accuracy, the adjustment of the receiving and transmitting terminals is done utilizing link maintenance control and tracking algorithm [26-27]. For most part, an impact observed named point ahead angular anisoplanatism is experienced for the condition PAA greater than isoplantic edge from tracking direction. Generally, it is brought about by the mismatch of the laser beam from the beacon path.

\subsection{Doppler Shift}

The variation in the recurrence of the received signal because of the relative movement among transmitter and receiver prompts Doppler impact. Occurring in inter-orbit satellite links where the satellite in lower orbits travel quicker than at higher orbits. Range of movement to be rapid via information hand off framework is $\pm 7.5 \mathrm{Ghz}$ for the transmission LEO and GEO, respectively.

The two LEOs can possess higher move engendering in inverse ways. The frequency move generated by Doppler around $140 \mathrm{kHz}$ move in a $2 \mathrm{GHz}$ clock time frame utilized for flagging. It requires a wide range of recurrence tuning for optical input filters or local oscillator (LO) lasers if there should be coherent optical link. This Doppler shift must be thought about for dependable FSO between satellite connections.

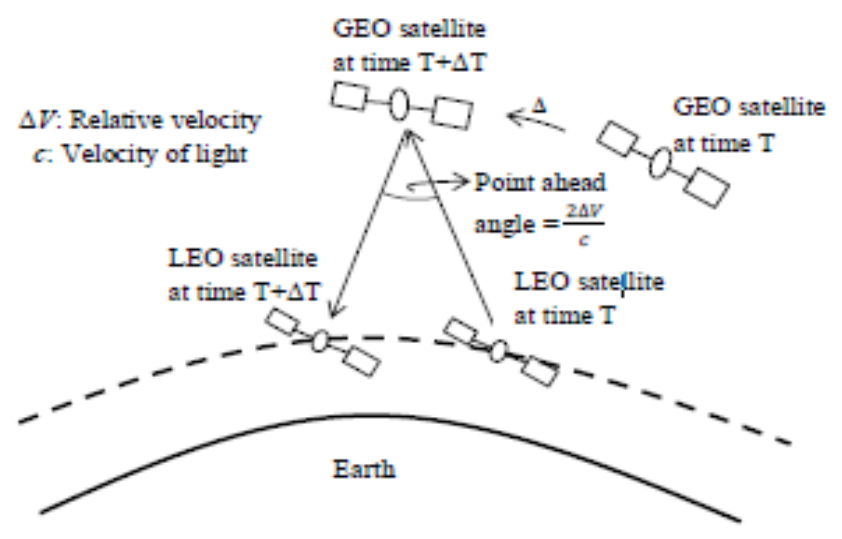

Concept of PAA in space communication

FIGURE 4. Concept of PAA in space communications. 
The Doppler shift achieves a greatest worth when the radial part of the relative speed achieves its most extreme. Carelessness of Doppler impact brings about loss of information and recurrence simultaneity issues at the beneficiary end. Incorporation of an optical phase lock loop (OPLL) procedure with the LO laser is needed for diminishing Doppler shift [28]. The agreeable recurrence tuning among the transmitters and LO at the collector is actualized to oppose the impact of Doppler shift. As far as a beat signal (distinction of transmitter and LO recurrence) is accessed, the recurrence procurement procedure starts with the adjustment of LO with an open recurrence control loop close to transmitter.

Recurrence tracking mode starts to lock on to the heterodyne recurrence control loop or homodyne loop, when the beat signal is retrieved. The utilization of the optical injection locking (OIL) method is other method; there is a downside that the locking recurrence range is constrained to commonly $1 \mathrm{GHz}$ or less [29]. Both OPLL method and OIL procedure i.e., utilization of an optical phase injection lock loop (OIPLL) strategy, that is used to lock the recurrence and phase under Doppler shift circumstances. A pilot-bearer coherent LEO to ground downlink utilizing the OIPLL Doppler recurrence move is shown in [30]. The impact of a Doppler move in homodyne inter-satellite communication link is investigated in [31]. Ideal transfer speed of the optical filter is resolved for the whole length of recurrence movements to improve the framework execution. The recurrence move prompted by Doppler impact is portrayed for LEO satellite constellation with optical inter-satellite links in [32]. For coherent homodyne recipient with optical phase lock loop the Doppler shift recurrence compensator is researched in [33].

\subsection{Satellite Vibration and Tracking}

The LOS signal is received by acquiring and tracking of the signals, such signals are created by the satellite or by the on-board laser communication sub-assembly. The above process of tracking such signals is a tough task for the FSO links. The different unsettling influences created externally due to the satellite are there because of the various aspects such as thrusters, momentum wheels, gimbal packages and solar panels, etc. There also exists various sources of noise caused by on-board laser communication system which comprises of Relative Intensity noise, background noise, dark current, shot noise, thermal noise, and signal shot noise. Satellite vibration and tracking issues and the discussion for restitution contemplated in [34].

\subsection{Background Noise Sources}

The inter-satellite link observes some noises whose origin rely upon the detection method, whether the system being pre-amplified or not optically. To detect the noise sources directly at the receiver's end, it is often observed that the noise formation is coming from bulk dark current this is the detector as well as the receiver amplifier. Which results in pre-amplifier noise and thermal noise and lastly the noise due to the signal i.e., shot noise. The local oscillator shot noise leads the system sources of the noise to detect coherently. Whereas, the stellar and celestial radiant fluxes results in the background sources of noise. 
The light dispersing from optics structure comprised with the light falling on the detector adds to the background noises.

\subsection{Acquisition, Tracking and Pointing (ATP)}

In FSO communication system pointing and acquisition of optical beam is a demanding concern in ground to satellite, inter satellite and satellite to ground links.

In [35] studied about the high-elevation airborne stages are connected to each other with the help of optical communications in free space. As a result, it developed as a promising answer for setting up remote systems for rustic and remote zones. The exhibition of FSOC framework is seriously corrupted due to the vacillation of angle of arrival (AoA) and pointing delusion. The arrangement of optical transmitter and recipient is accomplished by utilizing the pointing, acquisition, and tracking (PAT); however, the above structure should be compatible to work inside the space conquering of various challenges on dimensions, weight, and power. Because the platform of airborne is on the speedy shift, hence the PAT works quickly which is very alluring. As for the outage probability and for the most favorable beam dimension at the transmitter and receiver, the closed-form expression is allotted for this suggestion. For the précised derivation of the theory, the Monte Carlo simulations are used for the confirmation. The airborne FSOC system with fixed beam dimension above outspread radius of AOA variation and pointing delusion having the adjustable beam control strategy conquer the system.

In [36] have given an extensive overview on optical communications in free space use ATP structure. Provided mobile communications adopt ATP structure is a demanding fundamental. As for obtain line of sight, the proper adjustment of FSO transmitted receiver is needed and this is possible by using ATP structure. The classification of overviewed ATP structure is done as per their working assumptions, valued cases, exercised technology. The preferences and inconveniences of reviewed ATP components are recorded and talked about. Additionally talk about current difficulties and future research headings.

Pointing and acquisition of optical beam, turn into a primitive concern for the ground to satellite, inter satellite and satellite to ground FSO communication links. It happens because of the platform jitter disturbances, and narrow beam divergence of the optical beam [37]. Carrying a wide range of FOV, coarse detection is executed under the mode of acquisition. At the receiver end, there exists a detector which identifies based on the sensitivity of the position of the beacon signal. The receiver also continues to search for the required signal in its FOV side by side. While the presentation of the FSO link is constrained because of fluctuating pointing framework brought about by given stochastic systems: (i) following commotion made by the electro-optic tracker and (ii) vibrations made by inner satellite mechanical systems. The impact of satellite vibration and stage jitter is portrayed in [38]. The incorrect arrangement by any one may bring about connection disappointment or seriously debase the presentation of the framework.

The acquisition procedure is first tread for formation of FSO link and it includes the transmission scanning with its limited beacon signal over an ambiguity range. In large background radiations, the beam location by the receiver helped with beacon signal which is having adequate peak power and small pulse rate [39]. The once the guide signal is 
identified, the accepting terminal utilizes beam controlling components to point a relentless guide signal towards the starting terminal frequently balanced by fixed PAA. Figure 5 demonstrates the essential idea of ATP for a space FSO correspondence framework.

The telescopic assists in collecting the uplink signal from the ground level, which is further directed towards dichroic beam splitter-1. The splitter-1 will direct the coming signals to splitter-2 which later on guides the incoming signal to the ATP subsystem co-coordinating with signal's wavelength. Lastly, the beam splitter-3 converges the images from the initial situation on the focal pixel array (FPA). The area of this point on the exhibit speaks to the course from the received reference point signal in respect to the telescope's pivot (the focal point of the exhibit).

Which opt for the telescopic approach for the FOV? By the above approach the spatial securing procedure is carried out and on the other hand capturing the ground zero situation of the station on to the FPA. Finally, the signal is escorted from the satellite towards the receiver located at the base station thus shaping an interface for the LOS. Hence the reference point signal is obtained, governs the rational signal to taper down the FOV till the time the duo frameworks gets bolted among themselves. Hence, to start discovering the signal at the receiver satellite signal range request in the space, the signal range should be as per the requirement as well as the energy quotient (of the signal Obtained) should also be genuine to initiate the recurring circuit on its own. Regularly, the change from securing to following stage takes not exactly one second. At times, beaconless pointing utilizing divine reference source just is utilized to give an ideal pointing reference. This engineering furnishes a disentangled activity with extensive power sparing as it disposes of the need of additional reference point signal [40-42].

\section{Advantages of Is-OWC}

In the previous years, the Is OWC has turned out to be well known because of the succeeding advantages:

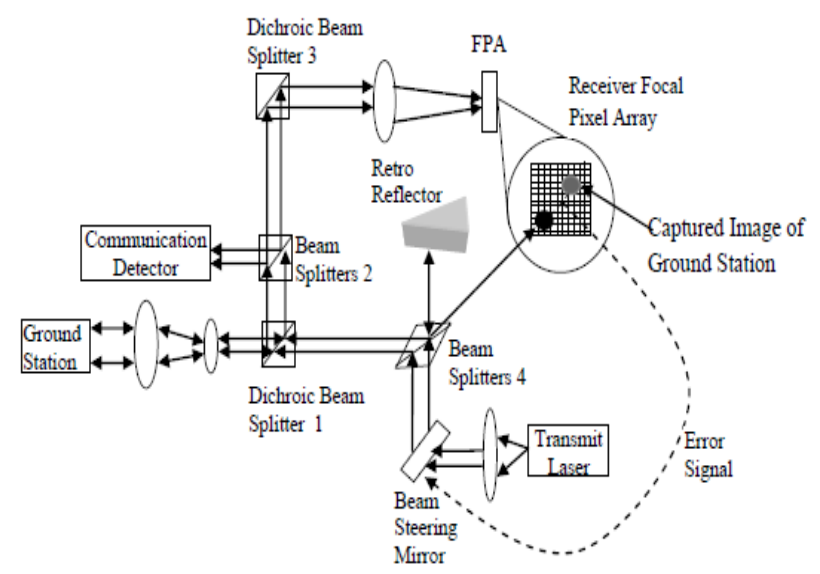

FIGURE 5. The ATP system between ground station and on-board satellite. 
1. High Data Rates: With the assistance of light as carrier frequency it is acceptable to be amplified using quick inter-satellite links [43]. Which flares up the information rates to Gbps to be used in the communication of the satellites optically.

2. Unlicensed Spectrum: The optical inter-satellite communication neither requires the necessity of license of spectrum nor for the co-ordination of the frequency as used in radio and microwave systems. Interference is certainly not a main task from or to system and furthermore, point-to-point laser signal is immensely difficult to intrude on, making it secure and safe.

3. Smaller Wavelength: The wavelength of light is significantly less with respect to microwaves. Signal wavelength of optical inter-satellite communication over RF/Microwave communication system is carrying huge variation. In this manner, RF/Microwave with respect to light wave describes the tapering nature of the thickness of the beam for respective magnitude of the signal [44].

4. Huge Bandwidth: The transmitting data in any communication system are associated with the modulated carrier bandwidth. Data transmission of around $2000 \mathrm{THz}$ is utilized as per the methods described for the high frequency optical carrier.

5. Reducing Antenna Size: The size necessity of the transmitter and receiver antenna is huge and furthermore massive if the RF/Microwave system is to be dynamic. While the antenna size gets diminished to centimeters just as its carrier frequency is enormous, in optical inter-satellite communication system. Thus, the satellite heaviness is reduced and power need is also decreased in optical inter-satellite communication system [45].

6. Small width beam dimensions: A small width beam is perceived for optical emission and its example a laser beam which is having diffraction restrained disparity in 0.01 to $0.1 \mu \mathrm{rad}$. This shows transmitting force is engaged with a thin range giving an optical inter-satellite link giving sufficient isolation from its probable interferers.

The different focal points incorporate insusceptibility to the radiofrequency interferences, faster communication, power efficiency, high accuracy or more all wireless optical communication does not contaminate the environment with electromagnetic radiations. All the subtleties determined are dynamic in an optical inter-satellite communication system since it can diminish the payloads and along these lines diminishing the cost.

\section{Conclusion}

As technology has advanced, methods of communication have additionally changed. Is-OWC is a phenomenal technology which has been generally utilized and investigated for terrestrial communication and broadcast purpose. As after contemplating the related work of this field, it is presumed that to accomplish more work on the different quality components like increase in bandwidth, transfer capacity, to upgrade the number of clients that can take an interest in the communication all the while. Thus further upgrades should be possible so as to evacuate these challenges discussed above by utilizing trendy mechanisms. Different advanced modulation formats can be utilized too for improving existing limit of the Is-OWC systems. 


\section{References}

1. Someda SG. Electromagnetic waves. Chapman \& Hall: Great Britain. 1998.

2. Karafolas N. Optical satellite networks. Journal of Light wave Technology. 2000, 18(12), 1792-1806.

3. Franck L. Routing in networks of inter-satellite links. IEEE Transactions on Aerospace and Electronic System. 2002, 38(3), 902-917.

4. Sharma A, Chaudhary S, Thakur D, Dhasratan V. A cost-effective high-speed radio over fiber system for millimeter wave applications. Journal of Optical Communications. 2017.

5. Upadhyay KK, Srivastava S, Shukla N, Chaudhary S. High-speed 120 Gbps AMI-WDM-PDM free Space optical transmission system. Journal of Optical Communications. 2017.

6. Heine F. Optical intersatellite communication operational. In: 2010 - MILCOM 2010 military communications conference. 2010.

7. Khalighi MA, Uysal M. Survey on free space optical communication: a communication theory perspective. Communications Surveys \& Tutorials. 2014, 16(4), 2231-2258.

8. Purvinskis RAJ. Interplatform links. University of South Australia. 2003.

9. Wood L, Ivancic W, Dörpelkus KP. Using light emitting diodes for inter-satellite links. In: Aerospace conference. 2010.

10. Chan VWS. Optical satellite networks. Journal of Light wave Technology. 2003, 21(11), 2811.

11. Djordjevic I, Ryan W, Vasic B. Coding for optical channels. Springer Science \& Business Media. 2010.

12. Bjarklev A, Chowdhury D, Majumdar A, Nakasawa M, Someda CG, Weber HG. Optical and fiber communications reports. 2008; 1-11.

13. Shekhar S, Gupta A. Inter-satellite optical wireless communication link (IsOWC). LAP Lambert Academic Publishing. 2015.

14. Chan VWS. Free space optical communication. Journal of Light wave Technology. 2015, 24(12), 4750-4762.

15. Moli-Sanchez L. Performance analysis of quantum cryptography protocols in optical earthsatellite and inter-satellite links. IEEE Journal on Selected Areas in Communications. 2009, 27(9), 1582-1590.

16. Dang A. Simultaneous acquisition and track scheme with multiple terminals based on subspace method for optical satellite networks. IEEE Transactions on Aerospace and Electronic Systems. 2010, 46(1), 263-277.

17. Ghosna FJ. Pulse position modulation coding schemes for optical inter-satellite links. Journal of IET. 2010, 46(4), 290-291.

18. Donner A. Satellite networks for aeronautical communication: traffic modeling and link load analysis. IET Communications. 2010, 4(13), 1594-1606.

19. Chatzidiamantis ND. Adaptive subcarrier PSK intensity modulation in free space optical system. IEEE Transactions on Communications. 2011, 59(5), 1368-1377.

20. Xie W. Received signal strength reduction analysis based on the wavelet model in inter-satellite laser communication. IEEE Journal of Light wave Technology. 2011, 29(15), 2327-2332.

21. Yang Y. Research bit error rate in the presence of local wave front aberration in inter-satellite laser communication. IEEE Journal of Light wave Technology. 2011, 29(19), 2893-2898.

22. Arruego I. In-orbit measurement of SET and DD effects on optical wireless links for intrasatellite data transmission. IEEE Transactions on Nuclear Science. 2011, 58(6), 3067-3075.

23. Smutny B, Lange R, Kämpfner H, Dallmann D, Mühlnikel G, Reinhardt M, Saucke K, Sterr U, Wandernoth B, Czichy R. In-orbit verification of optical inter-satellite communication links 
based on homodyne BPSK. Proc. SPIE, free-space laser communication technologies. 2008, 6877.

24. Smutny B, Kaempfner H, Muehlnikel G, Sterr U, Wandernoth B, Heine F. 5.6 Gbps optical intersatellite communication link. Proc SPIE, Free-space laser communication technologies. 2009, 7199.

25. Laser link offers high speed delivery. ESA Press Release. https://earth.esa.int/web/guest/news/-/ article/laser-link-offers-high-speed-delivery. Date accessed: 28/11/2014.

26. Ma X, Herbst T, Scheidl T, Wang D, Kropatschek S. Quantum teleportation over 143 kilometres using active feed-forward. Journal of Nature. 2012, 489, 269-273.

27. Zhou D, Refai HH, LoPresti PG, Atiquzzaman M. Control algorithm development for mobile FSO node alignment, In: IEEE/AIAA 28th digital avionics systems conference. 2009.

28. Guelman MKogan A, Kazarian A, Livne A, Orenstein M, Michalik H. Acquisition and pointing control for inter-satellite laser communications. IEEE Transactions on Aerospace and Electronic Systems. 2004, 40(4), 1239-1248.

29. Kahn JM. 1 Gbit/s PSK homodyne transmission system using phase locked semiconductor lasers. IEEE Photonics Technology Letters. 1989, 1 (10), pp. 340-342.

30. Lidoyne O, Gallion P, Chabran C, Debarge G. Locking range, phase noise and power spectrum of an injection-locked semiconductor laser. Proceedings of the Institution of Electrical Engineers. 1990, 137(3), 147-154.

31. Shoji Y, Fice MJ, Takayama Y, Seeds AJ. A pilot-carrier coherent LEO-to-ground downlink system using an optical injection phase lock loop (OIPLL) technique. Journal of Lightwave Technology. 2012, 30(16), 2696-2706.

32. Zhao F, Yu S, Ma J, Tan L. Effect of Doppler shift on differential phase-shift keying receivers using interferometric demodulation and balanced detection in intersatellite laser communication links. Optical Engineering. 2010, 49(10), 105001.

33. Yang Q, Tan L, Ma J. Doppler characterization of laser inter-satellite links for optical LEO satellite constellations. Optics Communication. 2009, 282(17), 3547-3552.

34. Ando T, Haraguchi E, Tajima K, Yamakawa S. Coherent homodyne receiver with a compensator of Doppler shifts for inter orbit optical communication. Proc. SPIE, free-space laser communication technologies. 2011, 7923.

35. Vuong V. Beam Size Optimization and adaptation for high-altitude airborne free-space optical communication systems. IEEE Photonics Journal. 2019, 11(2).

36. Kaymaket Y. A Survey on acquisition, tracking, and pointing mechanisms for mobile free-space optical communications. IEEE Communications Surveys \& Tutorials. 2018, 20(2), 1104-1123.

37. Arnon S. Power versus stabilization for laser satellite communication. Applied Optics. 1999, 38(15), 3229-3233.

38. Ho T. Pointing, acquisition, and tracking systems for free-space optical communication links. $\mathrm{PhD}$ thesis, University of Maryland. 2007; 1-220.

39. Koepf GA, Peters R, Marshalek RP. Analysis of burst error occurrence on optical intersatellite link (ISL) design. Optical Technologies for Communication Satellite Applications. 1986, 616, 129-136.

40. Hemmati H. Deep space optical communication. John Wiley \& Sons: New York. 2006; 683.

41. Nguyen TNT. Laser beacon tracking for free-space optical communication on small-satellite platforms in low-Earth orbit. Master's thesis, Massachusetts Institute of Technology. 2015; 1-94.

42. Boone BG, Bruzzi JR, Kluga B, Millard WP. Optical communications development for spacecraft applications. Johns Hopkins APL Technical Digest. 2004, 25(4), 306-315. 
43. Hindman C, Toberton L. Beaconless satellite laser acquisition-modeling and feasibility. In: Military communications conference. 2004; 41-47.

44. Chen H. Approach for earth observation satellite real-time and playback data transmission scheduling. Journal of Systems Engineering and Electronics. 2015, 26 (5), 982-992.

45. Uysal M. Optical wireless communications. Springer International Publishing: Switzerland. 2016. 